\title{
A Novel Half-Bridge Power Supply for High Speed Drilling Electrical Discharge Machining
}

\author{
He Huang, Jicheng Bai, Zesheng Lu, Yongfeng Guo
}

Department of Manufacturing and Automation Engineering, Harbin Institute of Technology, Harbin, Heilongjiang Province, China. Email: huanghe@hit.edu.cn, jichengbai@hit.edu.cn, lzesn@hit.edu.cn,guoyf@hit.edu.cn

Received February $10^{\text {th }}, 2009$; revised March $12^{\text {th }}, 2009$; accepted March $20^{\text {th }}, 2009$.

\begin{abstract}
High Speed Drilling Electrical Discharge Machining (HSDEDM) uses controlled electric sparks to erode the metal in a work-piece. Through the years, HSDEDM process has widely been used in high speed drilling and in manufacturing large aspect ratio holes for hard-to-machine material. The power supplies of HSDEDM providing high power applications can have different topologies. In this paper, a novel Pulsed-Width-Modulated (PWM) half-bridge HSDEDM power supply that achieves Zero-Voltage-Switching (ZVS) for switches and Zero-Current-Switching (ZCS) for the discharge gap has been developed. This power supply has excellent features that include minimal component count and inherent protection under short circuit conditions. This topology has an energy conservation feature and removes the need for output bulk capacitors and resistances. Energy used in the erosion process will be controlled by the switched IGBTs in the half-bridge network and be transferred to the gap between the tool and work-piece. The relative tool wear and machining speed of our proposed topology have been compared with that of a normal power supply with current limiting resistances.
\end{abstract}

Keywords: High Speed Drilling Electrical Discharge Machining, Half-Bridge Power Supply, Zero Current Switching, Zero Voltage Switching

\section{Introduction}

Electrical Discharge Machining (EDM), also known as spark erosion machining, is becoming increasingly popular. EDM sees the removal of matter from high hardness conductive materials by means of a series of repeated electrical discharges between electrode and work-piece, which are separated by a discharge gap. Dielectric fluid is forced into the discharge gap where electrical discharge erosion occurs. When a voltage large enough is applied, the dielectric fluid breaks down, the gap is ionized and electrons are emitted from the tool (cathode). When more electrons gather in the gap, the resistance drops, which causes electric spark to jump between the work-piece surface and the tool. The whole sequence of operation occurs within a few microseconds and is accompanied by a shock wave in the dielectric. The impact of the wave on the electrode causes high transient pressure. The current density in the discharge channel is of the order $10,000 \mathrm{~A} / \mathrm{cm} 2$. The temperature of the central point of the channel is of the order of tens of thousands of ${ }^{\circ} \mathrm{C}$. The forces of the electric and magnetic fields caused by the spark produce a tensile force and tear-off particles of molten and softened metal from this spot on the work-piece [1,2].

Within the scope of EDM, High Speed Drilling Electrical Discharge Machining (HSDEDM) is an important technology. The basic components of a HSDEDM system are as follows and the schematic is shown in Figure 1.

Like other EDM methods, the HSDEDM process is especially suitable for machining high strength and hard metal materials. Generally, a rotating thin copper or brass tube electrode is used as the drilling tool. High speed and pressure dielectric (water) is pumped through this hollow

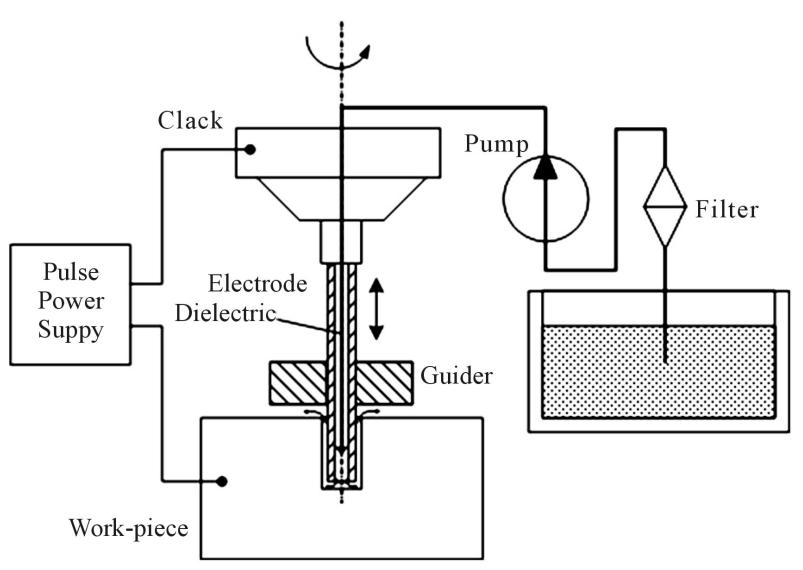

Figure 1. Diagram of HSDEDM process 
electrode and injected into the discharge gap to flush out eroded debris from working area to ensure the process remains stable. The dielectric fluid also acts as a coolant. Rotation of the electrode helps reduce deviation and uneven wear, which maybe otherwise be caused by the high pressure dielectric, on the face end of the electrode. The servo system acts to adjust the discharge gap and feed throughout the process. The most outstanding feature of HSDEDM is high speed of manufacture which can be up to $60 \mathrm{~mm} / \mathrm{min}$. This is hundreds of times the speed of the normal EDM and is exceeding the level of the traditional drilling. The processed apertures range from $0.3 \mathrm{~mm}$ to $3 \mathrm{~mm}$ and the aspect ratio can be up to 100 [3].

In HSDEDM, pulse power supply plays an important role in providing the thermal action of the electrical discharges between the electrode tool and the work-piece in order to achieve material removal. The Metal Removal Rate (MRR), the surface finish and Electrode Wear (EW) mainly depend on the magnitude and duration of the pulse discharge [4]. As the current increases, so do the MMR and the EW, although the surface finish decreases. As the discharge frequency is increased, the surface finish improves but the EW increases.

In order to improve the whole efficiency of the electronic equipment, the search for a more efficient switching technique has been developed. For a switch such as Insulated Gate Bipolar Transistor (IGBT), the zero current switching (ZCS) technology has been frequently used, because this technology can reduce the current rapidly when IGBT is turned off, therefore reducing the energy loss caused by the tail current [5,6]. It is also important, in miniaturizing power supply, to balance switching frequency and conversion efficiency. Zero Voltage Switching (ZVS) is a powerful means for increasing the switching frequency with small switching loss. On the other hand, switching noise is a critical problem at high frequency switching. ZVS is also effective in decreasing the spike current and surge voltage when switches are turned off [6].

This research has produced a pulse power supply that is used in HSDEDM system without an output capacitor for charging and discharging spark energy and bulky resistor for limiting current. A Pulsed Width Modulated (PWM) half-bridge topology with ZCS and ZVS is developed in this power supply for suppressing the noise current in discharge gap and surge voltage of switches.

\section{Developments in Power Configurations}

The shapes of voltage and current pulses in the discharge gap depend on the chosen power supply. There are three types of power supplies that have received most interest amongst the scientific community. They are Resistance Capacitance (RC) power supply (A), transistor switching circuit (B) and energy-saving power supply (C).

\subsection{Resistance-Capacitance (RC) Power Supply}

EDM was developed in Russia in the mid-1940-s by
Lazalenko and he used the basic RC power supply which is still used today in many cases when a fine surface finish is required [7]. The common configuration of these systems is shown in Figure 2.

The AC input voltage is normally fed into a variac and isolation transformer and is rectified to produce an uncontrolled DC. The bulk capacitor C1 is used to filter the rectified output and the output capacitor C2, which stores the energy needed for the spark, is connected in parallel to the discharge gap. Every time dielectric breaks down, the energy in C2 will be discharged and in the next cycle it will be charged again. The current is limited by the resistor, R1, which is normally a high power resistor. R1 is where most of the losses of an EDM power supply occur.

\subsection{Transistor Switching Circuit Power Supply}

Figure 3 shows a different technique of implementing the spark generator whilst utilizing the linear power supply. The transistor is Pulse Width Modulated (PWM) to control the total period of the charging time of the capacitor. This circuitry provides a higher MMR than the normal rectangular pulse power supply. In current research, more and more linear converters are being used to switch the electric energy. These improvements increase efficiency, power density and decrease the size of the magnetic components [1]. However this type of configuration still uses bulky resistors. The problem of high losses still remains.

\subsection{Energy-Saving Power Supply}

The power supply with the feature of energy-saving was developed in the end-1980s. Dr. Zhao Wansheng, with his group, developed a pulse generator for EDM with the feature. The schematic is shown in Figure 4. They designed the circuitry as a forward converter without the output capacitor. Current limiting is achieved by sensing the load current and feeding it back to the control circuit. Once the current reaches a present current limit, the controller shuts down the drive and starts again at next cycle. The forward converter topology is designed for use in low power (50W) to medium power (250W) applications [8]. The second winding of flyback transformer, T1, is used in the discharge circuit and acts as clamp winding. However this high inductive reactance will induce voltage swing and current tail in the discharge gap.

It can be seen from the developments in Power supply, the switch mode configuration and improvement of efficiency are research focuses in EDM.

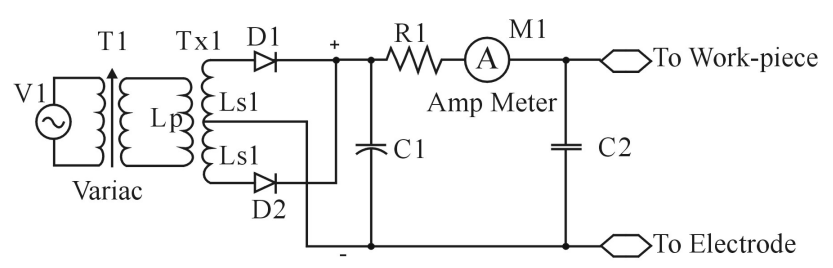

Figure 2. Traditional EDM power supply 


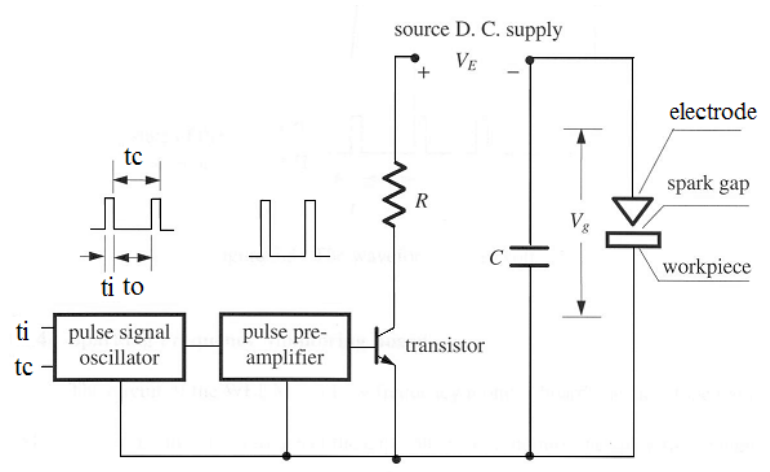

Figure 3. Transistorized RC power circuit

\section{Half-Bridge Network Power Supply}

The proposed topology is a PWM half-bridge converter, which uses IGBTs a as switch to control the energy. The configuration is shown in Figure 5.

\subsection{Configuration}

Two sets of IGBTs (TL1 to TLn and TR1 to TRn) are used as switches and connected with each other through the discharge side inductance $\mathrm{L}$, rd, discharge wires and discharge gap. L consists of the inevitable lead inductance, self-inductance in the discharge gap and any other stray inductances in the discharge path. The resistance of the discharge wires and the spark gap during discharge constitute rd. D1 and D2 are fly-wheel diodes. D3, D4, R3, R4, C1 and C2 constitute two sets of RCD snubber circuits which are used to protect the switches from voltage surge caused by the sudden turning off. Fast recovery diodes DL1 to DLn and DR1 to DRn are anti-parallel diodes in the switches which are also used to protect the IGBTs. In this way, ZVS of the switch is achieved even with high switching frequency.

\subsection{ZVS of Switches}

There are two sets of RCD snubber circuits parallel con-

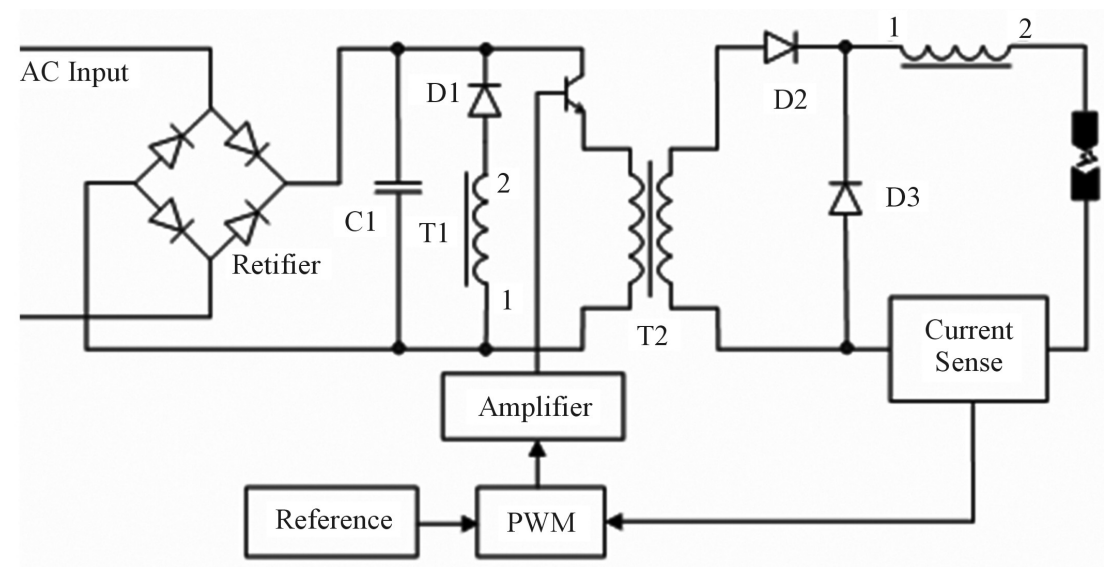

Figure 4. Energy-saving EDM power supply developed by Harbin Institute of Technology

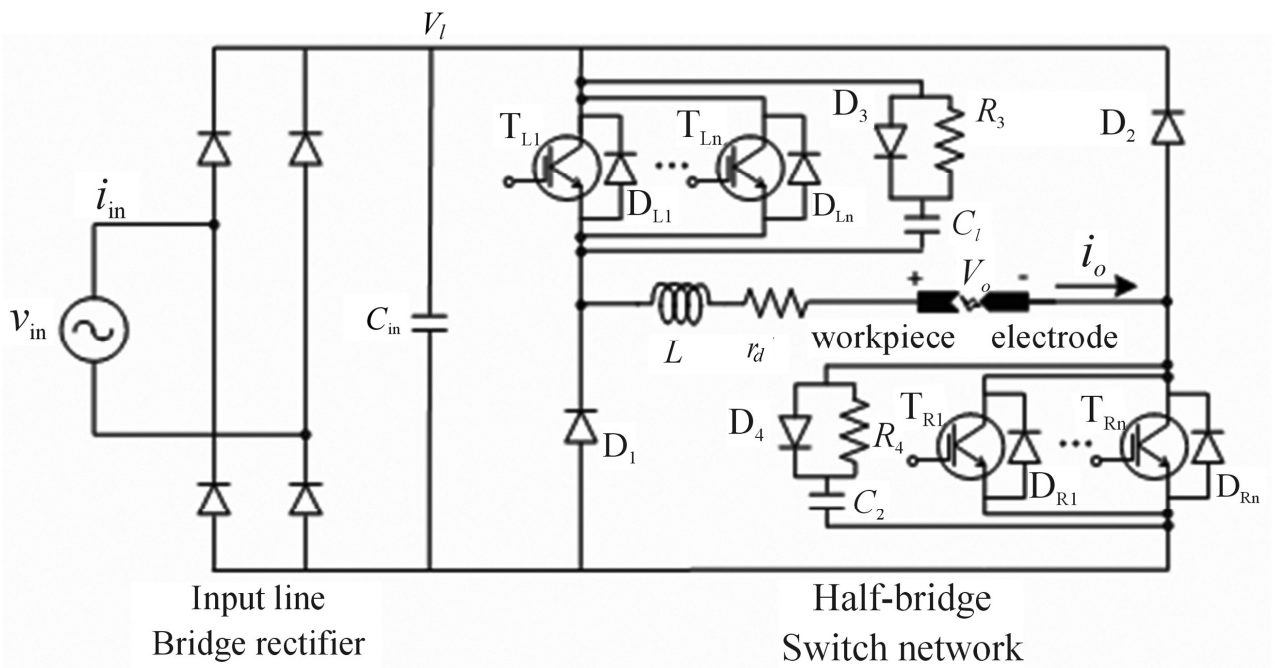

Figure 5. Circuit diagram of the HSDEDM power generator using a half-bridge network 
nected with switches as shown in Figure 5. The snubber circuits achieve ZVS for switches TLn and TRn. If without any protection measures for switch, when switch is turned off, the prime current $i_{p}$ decreases linearly, while the collector-emitter voltage $\left(V_{c e}\right)$ of the switch increases rapidly, and the surge voltage with extremely high value will be generated. In verify experiments, the surge voltage usually reaches more than six times of the supply voltage as shown in Figure 6(a). This surge voltage with high value is harm to switches. In this proposed topology, when switch is turned off, surge energy is stored in cap $\mathrm{C}_{1}$ or $\mathrm{C}_{2}$ through the fast recovery diodes $\mathrm{D}_{3}$ or $\mathrm{D}_{4}$. In the next time, when the switch is turned on, $\mathrm{C}_{1}$ or $\mathrm{C}_{2}$ discharge though the resistances $R_{3}$ or $R_{4}$. The measured waveforms of $V_{c e}$ and prime current of switch with ZVS can be seen in Figure 6(b).

\subsection{ZCS of Gap and Switching Methodology}

The traditional power supplies of EDM or HSDEDM always use bulky resistors to limit and control the current in the gap. The electrical energy surges also can be consumed by these resistors. For energy-saving, there are not current limit resistors in the proposed topology. If no action being taken to limit the power, the current will rise to a very high level to break the switches, especially when short circuit occurs. When the switch is off, the IGBTs also would be damaged by electrical surges caused by the sudden changes in electrical flow. In this PWM half-bridge network, when the drive IGBT is off, the fly-wheel diodes are used to smooth out the electrical surges caused by the sudden changes in electrical flow, therefore the ZCS can be obtained by this topology. This facilitates dielectric deionization quickly, therefore, short circuit and electric arc discharging are reduced and higher utilization of the pulse can be gained.

For this half-bridge network, the following PWM operational sequence is taken to generate the proper electrical pulses for the security of the switching IGBTs. The current in the discharge is shown in Figure 7.

- The left and the right IGBTs are all switched on in time t0. The power streams from source through the left IGBT, discharge wire, gap and the right IGBT to ground. The current in the gap rises rapidly to I1 which is safe for the switches.

- The left or the right IGBT is switched off in time t1. If the left IGBT is off, the energy stored in L streams through the circuit which consists of D1, wire, gap and the right IGBT to the ground. If the right IGBT is off, the energy streams though another circuit which consists the left IGBT, wire, gap and D2 and goes back to the source. In that time, the current decreases to I2.

- The step 1 and 2 are repeated several times. The on-time is t2 and the off-time is still t1. The current oscillates between I1 and I2. While the IGBT in left or right side is switched on and off repeatedly, the IGBT in the other side always is turned on.
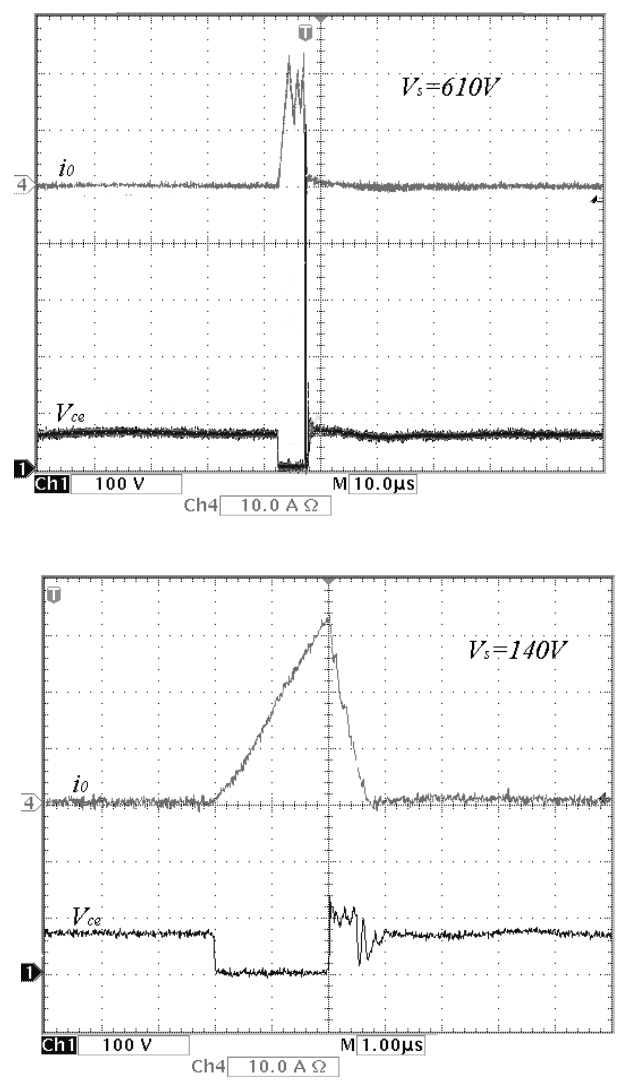

Figure 6. Waveforms of prime current in gap and $V_{c e}$ of switches

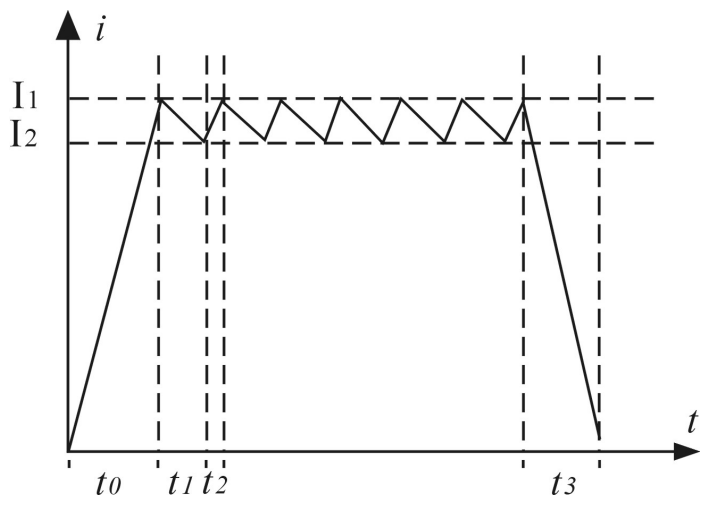

Figure 7. Theoretical current waveform in the discharge gap

- The left and the right IGBTs are all switched off. The energy stored in the wire inductance streams though flay-wheel diodes, D1 and D2 back to source. The current decreases rapidly to zero.

In Figure 7, a trapezoidal current waveform with saw-tooth form on the top can be seen. Compared with normal rectangular current waveform, the average current peaks is $(\mathrm{I} 1+\mathrm{I} 2) / 2$ and the width of the pulse is almost $\mathrm{t} 0+\mathrm{n}(\mathrm{t} 1+\mathrm{t} 2)+\mathrm{t} 3, \mathrm{n}$ is the repetition of switching on and off of IGBT just in left or right side. The peak current is depended on the simultaneous on-time (t0) of switches in 
both sides of the bridge in the beginning of the pulse. If just one IGBT is switched on in both sides in that time then t0 can not be set too large in order to avoid exceeding the rated current level of the IGBT. Larger peak current can be achieved by turning on more switches in both sides at the beginning of the pulse to help share the current. The width of the pulse is depended on number of the repetitions of switching. If only one IGBT is switched repeatedly in the middle of the pulse, the frequency is high and close to the rated frequency of the IGBT. This will lead to the increased transient thermal impedance of the IGBT, thus more heat generated by the current will damage the switch. In the proposed topology, several IGBTs are combined in parallel connection with collectors and emitters on each side. These IGBTs are switched by turns to decrease the working frequency of every one.

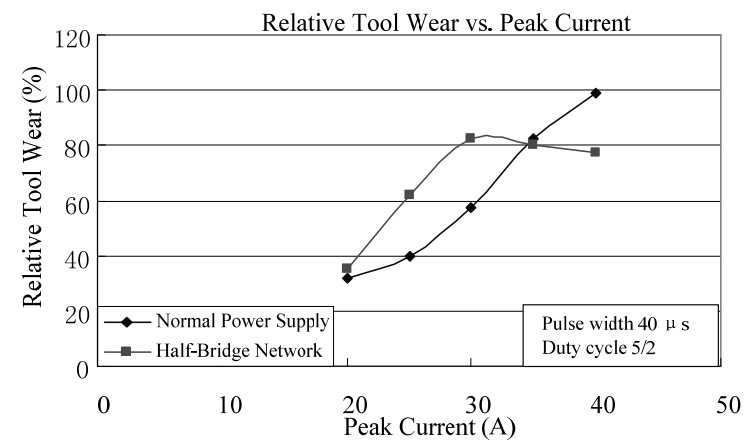

(a)

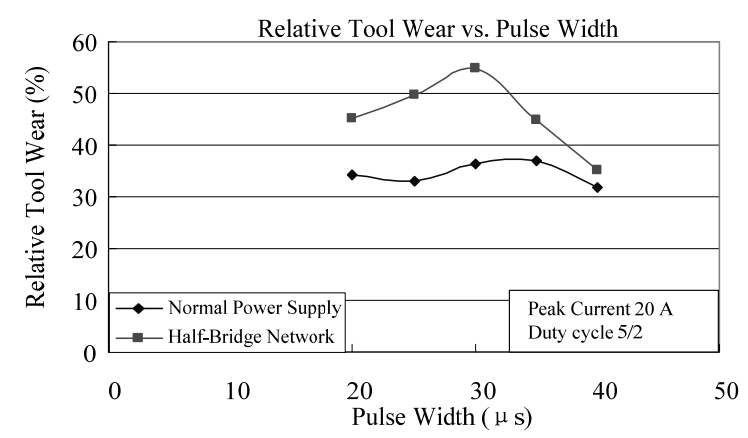

(c)

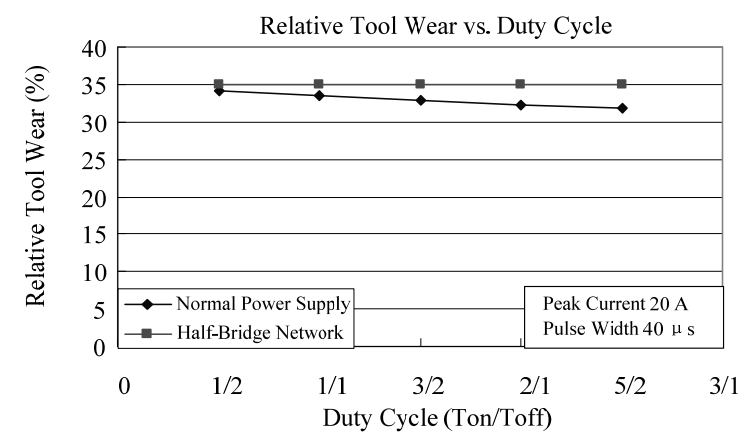

(e)

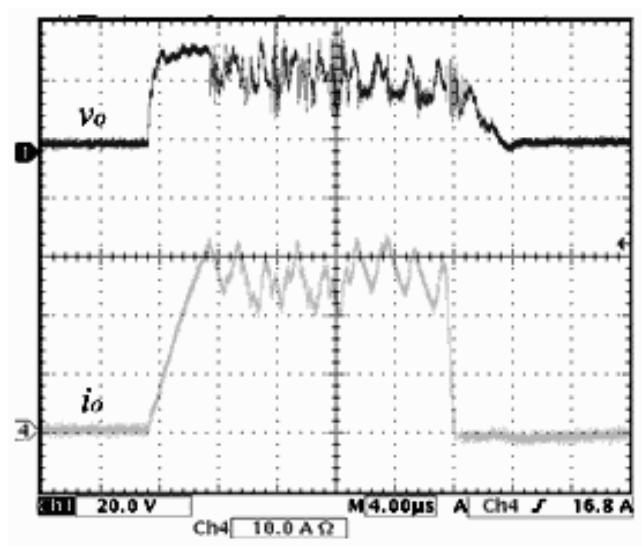

Figure 8. Experimental gap voltage and current waveforms

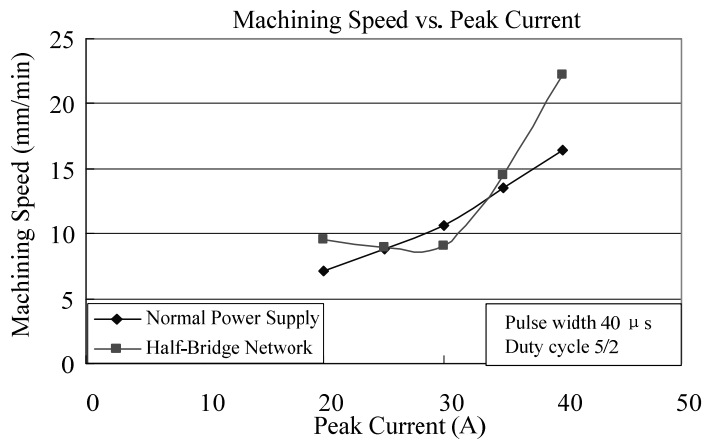

(b)

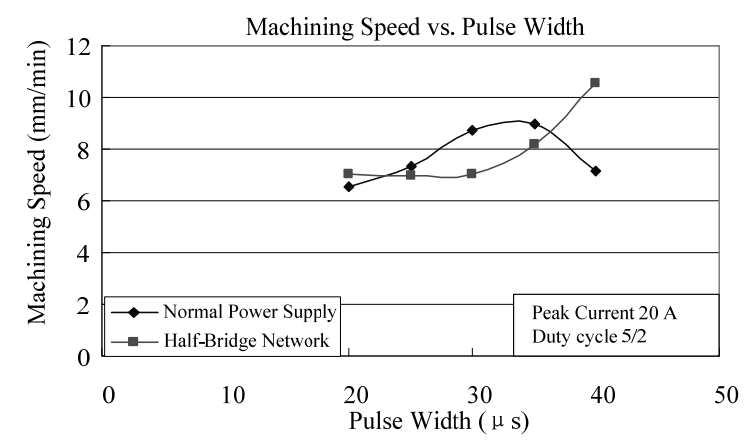

(d)

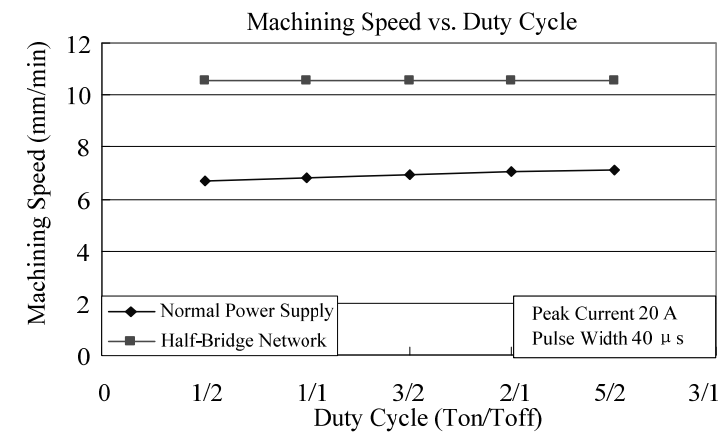

(f)

Figure 9. Relative tool wear and machining speed respond to 3 electrical parameters 
Figure 8 shows gap voltage and current waveforms of normal discharges. During the on-time the output current is around $25 \mathrm{~A}$ and the output maintaining voltage is around $20 \mathrm{~V}$.

It can be seen from Figure 8 that the power supply proposed in this research has good performance in controlling the energy in the discharge gap, even without current limiting resistor. The process features of the proposed power supply will be demonstrated by comparing with the normal power supply which using bulky resistor to limit current.

\section{Experimental Results}

Two types of HSDEDM power supplies were utilized to get relative tool wear and machining speed data. The first type is a normal power supply with current limiting resistances and the second type is the half-bridge network power supply without the resistances as proposed in this paper. Both types of the power supplies are characterized based on Relative Tool Wear (RTW) and EW data using three electrical parameters: gap peak current, pulse width and duty cycle. The comparison is shown in Figure 8. A brass tube electrode with $1 \mathrm{~mm}$ in external diameter and $0.3 \mathrm{~mm}$ in inner diameter was used in the experiments. The work-piece material was 40Gr steel and water was used as dielectric. The open voltage is $80 \mathrm{~V}$.

As shown in Figure 9, the proposed power supply achieves a higher machining speed in most situations, especially at larger electrical parameters. However, the relative tool wear achieved by using the proposed power supply is little larger than the normal one. In theory, the proposed power supply characters the improvement in electric energy efficiency, because the current-limiting resistances are removed. Detailed analyses of tool wear, improvement of efficiency and the methods to decrease electrode wear are future tasks.

\section{Conclusions}

A power supply for HSDEDM based on a half-bridge network without bulky current-limiting resistances in the discharge circuit was developed resulting in a great reduction in weight and size. A PWM operational sequence of switches was utilized to generate trapezoidal pulse waveform with saw-tooth form in the discharge gap. The electrical parameters of peak current, pulse width and duty cycle were achieved by control the switching time of these IGBTs in the circuit, This was also used to limit the current in the discharge circuit to avoid the power being too large to break the IGBTs. ZVS of switches was achieved to decrease the surge voltage when IGBTs were turned off. The "fly-wheel" diodes were developed to smooth the electrical surge and the ZCS technology was used to reduce the current rapidly when switches were turned off, therefore reducing the energy loss caused by the tail current. In comparative tests, it was shown that the normal power supply with current-limiting resistances has good performance when considering relative tool wear and machining speed at light loading conditions. However the machining speed of the normal one cannot compare to the performance of half-bridge network power supply at lager maximum poser outputs. This development paves the way for alternative in designing HSDEDM power supplies for high power applications.

\section{Acknowledgments}

This work was funded by National Science Foundation of China with the grant number: 50875064 and supposed in the frame of 863 research project of China with the grant number: 2007AA04Z345 and supposed in the frame of Heilongjiang province important science and technology fund with grant number: GA06A501. The authors would like to thank Y. L. Liu for help in data acquisition, G. Q. Deng and C. J. Li for preparing the devices, and A. Hird for invaluable advice concerning English writing.

\section{REFERENCES}

[1] C. M. F. Odulio, L. G. Sison, and M. T. Escoto Jr, "Energy-saving flyback converter for EDM applications," IEEE Region 10 Annual International Conference Proceedings, pp. 1-6, 2007.

[2] N. L. Kachhara and K. S. Shah, "The electric discharge machining process.” Journal of the Institution of Engineers (India) -Mechanical Engineering Division, No. 51, pp. 67-73, 1971.

[3] M. R. Cao, S. C. Yang, S. Q. Yang and Y. T. Qiao, “Experimental research on the process influencing machining velocity to small hole's EDM,” Modem Manufacture Engineering (in Chinese), Vol. 4, pp. 82-83, 2005.

[4] R. Casanueva, M. Ochoa, F. J. Azcondo, and S. Bracho, "Current mode controlled LCC resonant converter for electrical discharge machining applications,” Proceedings of the 2000 IEEE International Symposium on Industrial Electronics, Vol. 2, pp. 505-510, 2000.

[5] G. Hua and F. C. Lee, "Soft-switching techniques in PWM converters,” IEEE Transactions on Industrial Electronics, Vol. 42, pp. 595-603, 1995.

[6] K. Yoshida and T. Ninomiya, "A novel current resonant ZVS-PWM half-bridge converter,” Electronics \& Communications in Japan, Part I: Communications, Vol. 82, pp. 45-55, 1999.

[7] B. Sen, N. Kiyawat, P. K. Singh, S. Mitra, J. H. Ye, and P. Purkait, "Developments in electric power supply configurations for electrical-discharge-machining (EDM)," The 5th International Conference on Power Electronics and Drive Systems, Vol. 1, pp. 659-664, 2003.

[8] B. Y. Song, W. S. Zhao, and G. L. Shao, "Current type of electrical discharge machining pulse generator," China Mechanical Engineering (in Chinese), Vol. 12, pp. 386390, 2001. 